\title{
Evolving Solution Choice and Decision Support for a Real-World Optimisation Problem
}

\author{
Neil Urquhart \\ Edinburgh Napier University \\ 10 Colinton Road \\ Edinburgh, United Kingdom EH10 5DT \\ n.urquhart@napier.ac.uk
}

\author{
Achille Fonzone \\ Edinburgh Napier University \\ 10 Colinton Road \\ Edinburgh, United Kingdom EH10 5DT \\ a.fonzone@napier.ac.uk
}

\begin{abstract}
Agencies who provide social care services typically have to optimise staff allocations and the travel whilst attempting to satisfy conflicting objectives. In such cases it is desirable to have a range of solutions to choose from, allowing the agency's planning staff to explore the various options available This paper examines the use of multi-objective evolutionary algorithms to produce solutions to the Workforce Scheduling and Routing Problem (WSRP) formulated with three objectives which should be minimised: financial cost, $\mathrm{CO}_{2}$ emissions and car use. We show that financial cost and $\mathrm{CO}_{2}$ increase with the size of the problem and the imposed constraints. In order to support the planning staff in their decision making, we present an Evolutionary Algorithm based support tool that will identify a group of solutions from the Pareto front which match criteria specified by the planner. We demonstrate that our approach is able to find a wide range of solutions, which enhance the flexibility of the agencys choices, the decision support tool subsequently allows the planner to discover small groups of solutions that meet their specific requirements.
\end{abstract}

\section{KEYWORDS}

Evolutionary Algorithms, Transportation,Multi-Objective Optimisation, Decision Support, Real-World Problems

\section{ACM Reference format:}

Neil Urquhart and Achille Fonzone. 2017. Evolving Solution Choice and Decision Support for a Real-World Optimisation Problem. In Proceedings of GECCO '17, Berlin, Germany, July 15-19, 2017, 8 pages.

DOI: http://dx.doi.org/10.1145/3071178.3071207

\section{INTRODUCTION AND MOTIVATION}

Many real-world optimisation problems require solutions that optimise quantifiable constraints such as financial costs, distances travelled or $\mathrm{CO}_{2}$ produced. In such scenarios optimisation techniques can find a range of solutions (e.g. a non dominated Pareto Front), but there exists a layer of final decision making to be carried out by the planning staff. Where objectives conflict, there may be no single solution which is ideal in all aspects, for instance if environmental

Permission to make digital or hard copies of all or part of this work for personal or classroom use is granted without fee provided that copies are not made or distributed for profit or commercial advantage and that copies bear this notice and the full citation on the first page. Copyrights for components of this work owned by others than ACM must be honored. Abstracting with credit is permitted. To copy otherwise, or republish, to post on servers or to redistribute to lists, requires prior specific permission and/or a fee. Request permissions from permissions@acm.org.

GECCO '17, Berlin, Germany

(C) 2017 ACM. 978-1-4503-4920-8/17/07 ..\$15.00

DOI: http://dx.doi.org/10.1145/3071178.3071207 impact conflicts with financial cost, then there exists a level of political decision making required in order to determine which objectives should be prioritised and which should be compromised. This level of political decision making is undertaken by the planners based on factors such as political pressure, legislative pressure, public opinion, financial constraints or corporate and social responsibility policies.

In this paper we study a Workforce Scheduling and Routing Problem (WSRP), in such a problem, a group of employees must attend to a set of visits each of which must start within a specified time window and last for a specified duration. Each employee can perform more than one visit and employees are interchangeable. A solution takes the form of a schedule for each employee specifying which visits they should make and the order in which they should attend to them. The problem instances examined in this paper allow each employee to make use of car travel or public transport. The transport modes used determine the financial costs of the solution and its impact on environment and congestion.

We set out to answer two related questions, firstly can we generate Pareto sets of solutions that give the planner a meaningful choice and can we develop a mechanism to assist the planner when making the choice of which solution to adopt? The ability to give planners a choice of solution and to support them in making the decision as to which solution to adopt is necessary, if solvers based on techniques such as MOGAs are to gain acceptance in industry.

\section{PREVIOUS WORK}

The Workforce Scheduling and Routing Problem (WSRP) has been extensively investigated along with other related vehicle routing problems such as the Vehicle Routing Problem with Time Windows (VRPTW). Unlike vehicle routing problems, the focus of the WSRP is on individuals rather than vehicles; this focus allows WSRP researchers to examine aspects such as modal choice. For a comprehensive introduction to the WSRP and an overview of the latest developments, the reader is directed towards [2], [1] and [12]. The WSRP is closely related to the (VRPTW) which is studied in [21] using benchmark problem instances. The VRPTW has been formulate as a multi-objective problem in [3] using problem instances derived from real-world data. A number of previous researchers have dealt with problems relating to the scheduling and routing of workforces, [20] deals with home care scheduling, [17] with security personnel scheduling and [10] with technician scheduling. Comparison of different approaches to solve WSRP are difficult because no common benchmark problem instance has been defined so far.

To the best of our knowledge, the only work considering the travel mode within a WSRP type problem is [12], in which the authors investigate the scheduling of healthcare workers, the modes available 
to carers are public transport or private car. However, the travel mode of each employee is predefined and it cannot be changed in order to optimize the solution, also no account is taken of environmental impact. There have been a number of studies into green vehicle routing and logistics $[4,5,15]$. Of specific interest to this paper is [19], which focuses on the distribution of goods within the retail sector, as in this paper, the authors make use of real world data and emissions models, the problem scenarios are set within the GreaterLondon area and solutions are sought with low greenhouse gases emissions.

The first attempt to model the WSRP as a bi/multi-objective problem can be found in [1]. In that case, the authors use cost and patient convenience as the twin objectives. The authors define the solution cost as the travel cost and staff overtime costs. Patient convenience is defined as to whether the member of staff allocated is preferred, moderately preferred or not preferred, with penalties of 0 , 1 and 2 allocated as appropriate. The results presented show a strong relationship between convenience and cost; the more convenient a solution the higher the cost is likely to be. The authors also demonstrate that increasing the length of the time allocated windows (in practice relaxing the applied constraints) increases the likelihood of finding a less costly solution.

We note that previous research into the WSRP does not utilize environmental issues as a criteria, nor does it fully model the effect of allowing modal choice. To address these shortcomings, this paper formulates the WSRP as a multi-objective problem, with cost reduction, $\mathrm{CO}_{2}$ reduction and car use reduction as the criteria.

\section{METHODOLOGY}

\subsection{Problem Specification}

We evaluate the sustainability of a solution of the WSRP in terms of its $\mathrm{CO}_{2}$ emissions $(\mathrm{kg})$, financial cost (staff costs and travel costs in $£$ ) to the agency and percentage of trips made by car (considered as a proxy for the impact of the solution on traffic, and so on congestion and accidents). In the following, we refer to the environmental and car use objectives as external costs to differentiate them from the internal financial costs bourn by the agency. It may be expected that a larger use of public transport decreases the external cost but increases the internal one, because the reduction of working time due to longer travel times outperform the reduction of in travel costs. This expectation is valid in contexts where the door-to-door duration of journeys by public transport is on average longer than that of car-based journeys. This may not be true in very congested cities with priority measures for public transport. A solution of the WSRP as defined in this paper includes the transport mode used by employees, either a car or public transport. Car journey paths and times are determined using the GraphHopper library [16] based on OpenStreetMap $[9,11]$ data, public transport times and costs are derived from the Transport for London (TfL) API [8] The costs associated with each solution are calculated as follows:

- CO2 emission: for cars we adopt the WebTAG UK transport assessment model. For public transport we use the emission factors published by TfL [7].

- Labour cost: the median of the hourly salary of a social worker in the UK is $£ 16$ [18] and, according to EU official statistics, the wage is around $80 \%$ of the labour cost for social work activities. Hence we assume a labour cost of $20 £ / \mathrm{h}$. We consider daily shifts of maximum $8 \mathrm{~h}$.

- Travel cost: we cost journeys assuming a reimbursement rate of $£ 0.56$ per mile [6] for passengers public transport, we assume a daily travel cost of $£ 11.80$, based on TfL daily ticket costs.

The values of internal and external costs clearly depends on the number of visits, the spatial distribution of the visits in relation to the point from which the employees start their daily shift, the duration of each visit and the duration of the time window in which each visit has to begin. We explore the relation between these factors and the costs of the mobility required by non-residential care services by means of synthetic sets of visits, with random locations in the City of London. We assume that all the carers begin their daily shift from the agency headquarters. We analyse the costs of mobility considering four cases, representing different operational conditions (workload and spatial distribution of the visits) of the agency:

- London: 60 visits at randomly selected locations, within London, each visit being within a radius of approximately 16 miles of the agency headquarters.

- BigLondon: 110 visits based on the 60 visits and agency headquarters from the London data, with an additional 50 random visits within a radius of approximately 23 miles of the agency headquarters.

- Offset: 110 visits set out as per BigLondon, but with the headquarters located on the outside of the visit cluster.

- Cluster: 60 visits grouped in six clusters. The clusters are randomly distributed around the headquarters.

For each operational case, we consider six types of service provision, represented by adding one of the following identifiers to the name of the operational condition:

- 1: all visits have a time window of 8 hrs. i.e. the visits can start any time during the working hours of the employees.

- 2, 4, 8: Each visit has a 4, 2 or $1 \mathrm{hr}$. time window allocated. The beginning of the time window is chosen randomly.

- Rnd: The duration of the time window of each visit is randomly chosen among 1, 2, 4, or 8 hrs.

- R00: Visits have $1 \mathrm{hr}$. time window, with a duration of 15, 30,45 or $60 \mathrm{~min}$ randomly allocated. With the exception of R00, the duration of each visit is 30 minutes.

Data files representing the problem may be found witin the supplementary material accompanying this paper, the results files containing the Pareto fronts used within the solutions are available from [22].

\subsection{Solution Generation}

The approach taken, based upon that described by the authors previously [23, 24] uses a portfolio of Multi-Objective Evolutionary Algorithms (MOEAs), which produce a set of Pareto optimal (nondominated) solutions. MOEAs have been proven to find good solutions quickly in complex environments including those that are $n p$ hard - although there is no guarantee that they will find the optimal solution. For many real world problems it is not necessary to find the optimal solution, but only to find a good-enough solution. The difference in practical terms (such as costs, emissions or times) between good enough and optimal salutations may be small enough 
so as not to make any practical difference (e.g. a small financial saving). Given that the costs used within the optimisation process are estimated they may differ from the costs generated by an implemented solution - e.g. unexpected traffic congestion or public transport delays could increase $\mathrm{CO}_{2}$ produced or financial costs, thus negating the effects of choosing a solution with a very small cost advantage over a solution found earlier in the process.

All of the algorithms within the portfolio use the same basic problem representation, this being a permutation of visits and travel modes between visits for all of the employees and visits in the problem, when constructing a solution each visit is added to the solution in the permutation order. For the first visit, there will be no employees within the solution so the visit is added as the initial visit of a new employee, the travel mode for that employee being determined by this visit. Each subsequent visit in the chromsome is then considered, being added to the first employee that feasibly undertake the visit using matching travel mode from their previous visit. If the visit cannot be added to any of the existing employees, a new employee is created and the visit is added to that employee. In this way a feasible solution is constructed from the problem representation. When a solution has been built, its internal costs, $\mathrm{CO}_{2}$ emission and impact on traffic may be calculated making it possible to identify those genotypes that encode better solutions.

\subsection{Solution Evaluation}

Given the tri-dimensional nature of our problem, we visualize the Pareto sets using plots of parallel coordinates, a common technique for showing points in $\mathrm{n}$ dimensional space. It is necessary to scale each of the objectives used in the range 0 to 1 , where 0 represents the lowest value found for that objective and 1 the highest value found. The scaled performances of each solutions are then interpolated to show the relationships and to compare different solutions (see figure 1). Within each plot, each axis represents one dimension and each polyline represents one solution, showing its position on each of the three axis. For a full review of the use of parallel coordinates and multi-dimensional problems, the reader is referred to [14].In the plots presented in this paper the best and worst solutions, for each objective, are highlighted to allow the reader to gain a further insight into the trends.

We utilize also the Hypervolume metric [25] which gives an indication as to the overall quality of a Pareto front. Each solution may be plotted in 3-dimensional space using normalized objective values. In this representation, the solution space is bounded by the points $(1,1,1)$ and $(0,0,0)$. Hypervolume measures the volume between the Pareto front and the vertex $(0,0,0)$ of the hypercube defined by the abovementioned bounding points; the larger the Hypervolume the greater the number of solutions dominated by the front. Using normalized objectives, the Hypervolume can assume values in the range $0-1$, a volume of 0 represents a single solution with the worst values in all objectives, a volume of 1 represents a solution with the best observed values in all three objectives (a solution that would dominate all solutions produced thus far). Therefore, the hypervolume metric allows us to compare two Pareto fronts in order to evaluate which represents better progress on the objectives.

\subsection{Visualisation of the Pareto Set}

When a Pareto front is produced, it is useful to have some form a visualisation in order to determine the size of the front and the range of the objectives covered. For a 2-dimensional problem the front can be plotted by allocating each dimension to the $\mathrm{X}$ and $\mathrm{Y}$ coordinates, the front can then be plotted as a curve. For dimensions greater than two, plotting becomes problematic - a 3 dimensional problem may be plotted as a surface, but visualisation, especially when rendered in $2 \mathrm{D}$ can be difficult. For multi-dimensional problems the technique of parallel coordinates [14] allows many solutions to be visualised on one plot and the relationships between the solutions themselves and the objectives to be explored.

For each objective within a parallel coordinates plot a vertical axis is created, a solution is then represented by a polyline liking the axis together at the points appropriate for that solution. The parallel coordinates visualize the relationships between the objectives. By using four axis, each relationship between each pair of variables is shown.

\subsection{Supporting the Choice of Solution}

The Pareto front produced by the MOGAs within the portfolio may contain several hundred solutions which are all non-dominated. This set of solutions represents only a small fraction of the size of the total search space. We are presented with the practical problem that the agency planner still has to choose a single solution to adopt. Whilst we can visualise such multi-dimensional Pareto fronts using parallel coordinates (see section 3.4) the plot is often too crowded to allow individual solutions to be picked out. The practice of "brushing" [14] allows the user to highlight sections of one or more axis in order to highlight only those polylines that pass through the highlighted sections. Brushing can allow a user to explore the solutions within a crowded parallel coordinates plot [13] which goes some way towards supporting the decision making process.

We develop a tool which will highlight a few members of the Pareto front which may be of particular interest to the user. We adopt two principles, firstly that we allow the planner to specify areas of interest to them (in a manner similar to brushing) and that we allow the planner to specify the minimum distance that significantly separates two solutions on an axis. Whilst the MOGA may produce a Pareto front that contains, for example, solutions with a financial cost of $£ 5$ and $£ 6$ or $\mathrm{CO}_{2}$ values of $42 \mathrm{~g}$ and $43 \mathrm{~g}$, reality might suggest that the difference between the estimated costs in the solver and the real-world costs of the adopted solution are such that such small differences are insignificant when making the final choice. We can allow the user to specify what the significance value is and then eliminate solutions that are deemed to not be significantly different.

Our solution to this problem, allows the planner to specify ranges of interest for each axis and minimum differences for each axis if no criteria are specified then the axis is not filtered. A simple evolutionary algorithm is adopted, the representation being a binary string, one bit for each member of the Pareto front, a 1 signifies that the solution is filtered a 0 signifies that the solution is to be left in. A population size of 20 is utilised, in each generation we create one child using uniform crossover and a random bit flip mutation. An outline of the EA is given in algorithm 1 and the parameters used in table 1 . 


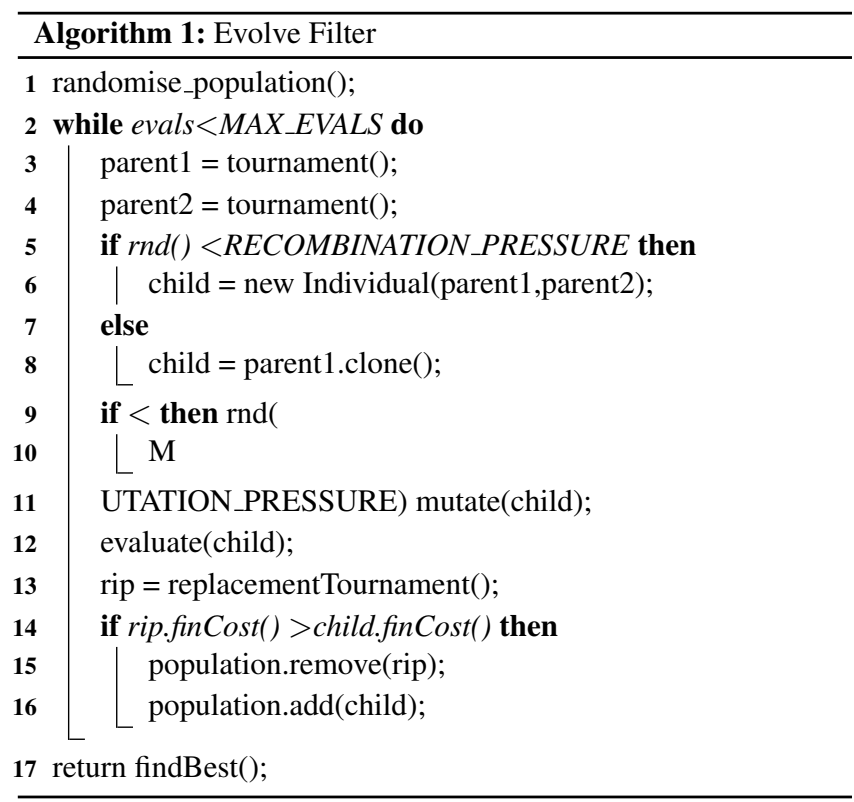

Table 1: Parameters used within the Evolutionary Algorithm

\begin{tabular}{|l|l|}
\hline Parameter & Value \\
\hline Population size & 20 \\
\hline Recombination Pressure & 0.5 \\
\hline Mutation Pressure & 0.5 \\
\hline Selection tournament size & 2 \\
\hline Replacement tournament size & 2 \\
\hline
\end{tabular}

The fitness function examines each solution and adds penalties as follows:

(1) Any solution not filtered on an axis that is out of range add a penalty of the difference between the item value and the closest range limit

(2) Any solution that is filtered, but lies within a range then add a fixed penalty of 0.01

(3) Any pair of solutions on an axis that are closer together than the specified criteria add a penalty based on the actual difference, less the minimum specified

The first two items penalise solutions which are unfiltered outside of specified ranges and filtered within specified ranges, the second item is required to ensure that the EA is rewarded for including solutions that meet the users' criteria. Items 1 and 3 give graduated penalties which increase depending on the severity of the issue. The criteria that the user may specify for any of the axis is given in table 4. Note that because the filtering is carried out after the generation of the initial Pareto set, filtering criteria can be applied to any of the axis on the parallel coordinates chart and not just those objectives used in the initial optimisation. Currently the algorithm can undertake 400,000 evaluations in approx. 10s (coded in Java on an MS-Windows PC), which allows it to be used to support rapid decision making, the planner can try many different sets of criteria quickly to explore the solutions available.

\section{RESULTS}

\subsection{Solution Range}

The algorithm portfolio was applied to the problem instances described earlier and the solutions were characterized in terms of $\mathrm{CO}_{2}$ emissions, total cost and \% of employees using cars, a Pareto front of non-dominated solutions was produced for each problem, see table 2. The size of the Pareto front (i.e. the quantity of non-dominated solutions) ranges between 119 and 942, representing a large number of non-dominated choices available to the decision-maker. Figure 1 gives an indication as to how evenly distributed these solutions are within each objective. The position of the solutions within each axis highlights areas where solutions do or do not exist. For instance figure 1d shows that for the offset data set, there are few solutions in the fronts that have low car use, $0 \%$ car use solutions exist, but there is solutions between 0 and $30 \%$. The figures show that gaps such as that are rare, so if the decision-maker has a specific target to meet for one of the objectives (e.g. low $\mathrm{CO}_{2}$ ) then they are likely to find a solution that meets that requirement.

Table 2 shows a relation between the quantity of solutions found and the problem constraints, represented by the time windows. Times can be allocated to visits in a more flexible way in problem instances with larger time windows (see the problem specification), such flexibility allows finding solutions that are at the same time more sustainable and less expensive for the agency. It follows that the less constrained problems have smaller Pareto fronts, because it is easier to find quasi-global optimal solutions, i.e. solutions that dominate more solutions of a lesser quality. As expected, the quantity of available solutions increase with the number of visits. On the contrary, it decreases when the visits are organised in clusters, i.e. clustering visits is equivalent to make the problem more constrained. The possibility of pursuing sustainability clearly depends not only on the size of the front but also on the value of the absolute performances. Both $\mathrm{CO}_{2}$ emissions and total costs increase when the problem is more constrained or of bigger size, i.e. in those cases it is easier to find solutions as mentioned above, but such solutions are less optimised. The conclusion is confirmed by the hypervolume metric, which tends to be inversely related to the size of the front. Note that the increase in total cost is related to the increase of the number of staff in each solution. Clearly this number rises as the problems become more constrained, because with shorter time windows, a greater quantity of visits have to take place concurrently. Two visits that take place concurrently requires two members of staff, whereas if the visits can be made sequentially it may be possible for one member of staff to undertake both of the visits.

Table 3 shows typical examples of the best individual solutions obtained for each problem instance. Assuming the agency wishes to explore the effect of minimising a specific objective, we present details of the solution found that represents the best performance in that objective. Note that the car use objective is largely binary, with solutions featuring $100 \%$ car use being consistently found for the lowest cost solution. Where car use is the target, solutions with $0 \%$ car use are also consistently found. When targeting $\mathrm{CO}_{2}$ minimisation solutions featuring $0 \%$ car use are found in every case except for the more constrained offset problems.

We note that the solutions showing lower costs tend towards higher $\mathrm{CO}_{2}$ levels, and feature $100 \%$ car use. This may be explained 
Table 3: Examples of solutions found when minimising each of the objectives and examples of the compromise solutions

\begin{tabular}{|l|l|l|l|l|l|}
\hline Problem & Target & CO2 $\mathbf{( k g})$ & Total Cost $(\mathfrak{(})$ & Car Use & Staff \\
\hline lon-4 & Cost & 274.0 & 865.3 & 1.000 & 8 \\
\hline lon-4 & CO2 & 109.9 & 1522.0 & 0.000 & 10 \\
\hline lon-4 & CAR & 119.2 & 1493.3 & 0.000 & 12 \\
\hline lon-4 & Comp. & 271.0 & 865.9 & 1.000 & 8 \\
\hline cluster-2 & Cost & 326.0 & 1003.9 & 1.000 & 7 \\
\hline cluster-2 & CO2 & 203.6 & 1620.8 & 0.000 & 11 \\
\hline cluster-2 & CAR & 203.6 & 1620.8 & 0.000 & 11 \\
\hline cluster-2 & Comp. & 315.0 & 1011.7 & 1.000 & 7 \\
\hline blon-1 & Cost & 556.0 & 1735.0 & 1.000 & 10 \\
\hline blon-1 & CO2 & 384.3 & 3093.7 & 0.000 & 18 \\
\hline blon-1 & CAR & 393.3 & 3079.1 & 0.000 & 18 \\
\hline blon-1 & Comp. & 501.7 & 2866.0 & 0.235 & 17 \\
\hline offset-8 & Cost & 1148.0 & 2489.8 & 1.000 & 19 \\
\hline offset-8 & CO2 & 874.1 & 3579.3 & 0.038 & 26 \\
\hline offset-8 & CAR & 961.0 & 3588.6 & 0.000 & 27 \\
\hline offset-8 & Comp. & 1080.0 & 2544.0 & 1.000 & 19 \\
\hline
\end{tabular}

by the average time for a public transport journey being 77 minutes, a car journey being 37 minutes. Thus solutions which make greater use of public transport incur greater staff costs due to the increase in staff time spent travelling. Conversely when targeting the $\mathrm{CO}_{2}$ objective, 15 of the 24 solutions produced feature $0 \%$ car use, and in the remaining solutions the highest car usage is $11.5 \%$. Since it is necessary to make more use of public transport, which in turn requires more staff hours, internal costs increase. The average internal cost of a solution, (across all problem instances) that targets $\mathrm{CO}_{2}$, is $£ 2626.5$, the average cost of one that targets cost is $£ 1566.7$ and the average cost of equivalent compromise solutions is $£ 1878$. As well as the three objectives, the number of staff required by each solution is also shown, this property being of considerable interest to the agency. We note that when targeting costs, fewer staff are required by the solution, as it can be expected since table 2 shows that salary accounts for around $90 \%$ of the total costs of the solution.

The relations between the objectives, outlined above, may be viewed in the parallel coordinates shown, examples of which are shown in Figure 1, Due to space constraints, we only reproduce a small selection of the charts in this paper, but interactive plots may be found online at http://www.soc.napier.ac.uk/ 40000408/ results/parallelcoords.html . Within each of the plots, four solutions are highlighted, The red polyline highlights the solution with the lowest $\mathrm{CO} 2$, green the lowest total cost, red the lowest car use, the compromise solution (closest to the average in each objective) is highlighted in black.

Figure 1a (London-2) shows a typical relation between $\mathrm{CO} 2$ and total cost, with lower $\mathrm{CO} 2$ generally corresponding to higher total costs. However, the pattern of the polylines suggests that it is not a strong correlation. A stronger relationship is noted between total costs and car use with the crossing points contained in a much smaller area. The relationship between car use and $\mathrm{CO} 2$ is fundamentally different, the polylines are far closer to being parallel and no central crossing point emerges. This confirms that car use and $\mathrm{CO} 2$ have a strong relation, as expected, with high car use likely to be associated with high $\mathrm{CO} 2$, and vice versa.

Figure $1 b$ and $c$ show the same fundamental relationships as Figure 1a, but significantly Figure 1c has a gap at the lower end of the cost axis, demonstrating a lack of solutions with lower costs. Figure 1d shows an example of a problem where the fundamental relation between $\mathrm{CO} 2$ and total cost has changed. The visualisation suggests a poor correlation between $\mathrm{CO} 2$ and total cost although
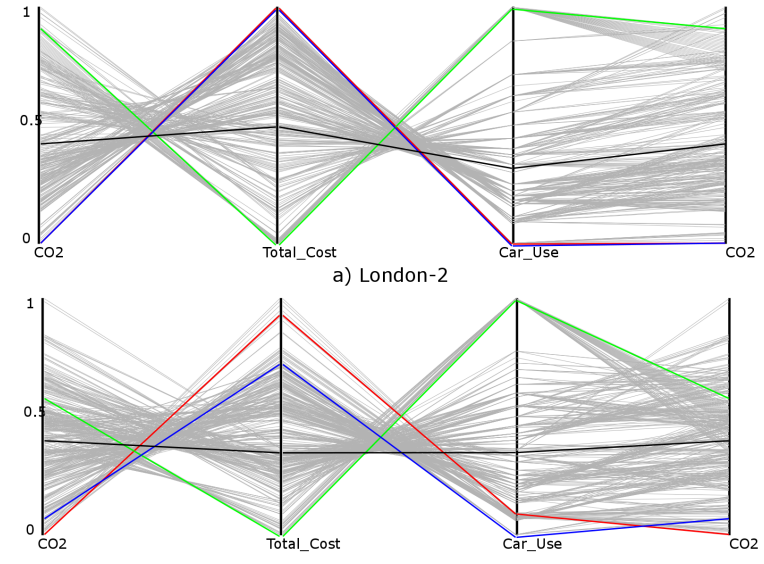

b) Cluster-rnd

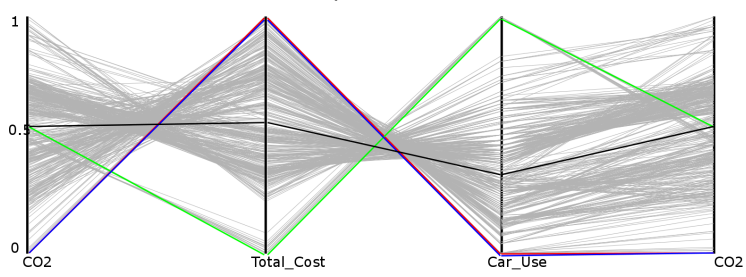

c) BigLondon-rnd

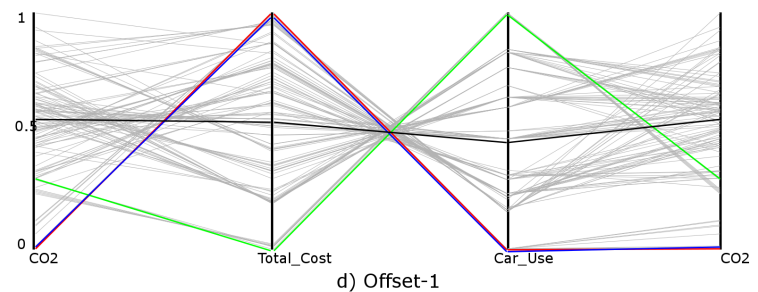

Figure 1: Parallel coordinates plots for a range of Pareto fronts. The Solution with the lowest $\mathrm{CO} 2$ is denoted by the red polyline, the green denotes the lowest Total Cost and the blue the car use. The compromise solution is highlighted in black.

these variables have a stronger correlation in other problems (as demonstrated in Figure 1a).

Overall, Figure 1 and table 2 show that, within the general trends suggested by the analysis of table 3 , our approach allows fine-tuning solutions which may satisfy the generally clashing objectives of promoting sustainability and reducing total costs of the provider of the care service.

\subsection{Supporting Decision Making}

We apply the decision tool [22] to a set of Pareto fronts produced using the portfolio technique, with $\mathrm{CO}_{2}$, total cost and car use as the criteria, tables 5, 6,7 and 8 show the results of applying four randomly selected sets of criteria to Pareto fronts generated for each of the problems discussed earlier. We note that there are a number of scenarios where the filter produces no results, there may well be situations where there are no solutions in the initial Pareto set that match the criteria specified, in such cases no results are returned. Relatively small numbers of solutions are returned in most cases, the highest average being 8.4 (table 8 , thus the planner 
Table 2: A summary of the results obtained. The objectives and solution characteristics represent average values across all of the solutions in the front.

\begin{tabular}{|c|c|c|c|c|c|c|c|c|c|}
\hline \multirow[b]{2}{*}{ Operational conditions } & \multirow[b]{2}{*}{ Service type } & \multicolumn{2}{|c|}{ Pareto Front Characteristics } & \multicolumn{3}{|l|}{ Dimension } & \multicolumn{3}{|c|}{ Costs to the agency providing the service } \\
\hline & & Hypervolume & Size & Total Cost $(f)$ & $\mathrm{CO} 2(\mathrm{~kg})$ & Car Use & Staff & Staff $\operatorname{cost}(f)$ & Travel Cost $(f)$ \\
\hline \multirow[t]{6}{*}{ London } & 1 & 0.761 & 229 & 1068 & 144.8 & 0.42 & 6.9 & 984.7 & 83.1 \\
\hline & 2 & 0.704 & 315 & 1166 & 163.8 & 0.35 & 9.3 & 1057.9 & 108.4 \\
\hline & 4 & 0.618 & 271 & 1188 & 215.5 & 0.39 & 12.8 & 1041.5 & 146.3 \\
\hline & 8 & 0.533 & 473 & 1270 & 247.3 & 0.38 & 15.8 & 1095.4 & 174.4 \\
\hline & r00 & 0.370 & 511 & 1546 & 263.2 & 0.39 & 18.1 & 1357.4 & 188.3 \\
\hline & rnd & 0.632 & 338 & 1175 & 211.1 & 0.42 & 11.0 & 1043.3 & 132.2 \\
\hline \multirow[t]{6}{*}{ Cluster } & 1 & 0.758 & 174 & 1197 & 225.9 & 0.43 & 7.8 & 1090.9 & 105.7 \\
\hline & 2 & 0.586 & 279 & 1315 & 286.3 & 0.41 & 10.8 & 1170.3 & 144.5 \\
\hline & 4 & 0.520 & 267 & 1477 & 284.5 & 0.29 & 14.3 & 1305.9 & 171.0 \\
\hline & 8 & 0.468 & 467 & 1452 & 328.3 & 0.38 & 16.6 & 1266.9 & 185.5 \\
\hline & $\mathrm{r} 00$ & 0.341 & 463 & 1715 & 326.2 & 0.36 & 17.5 & 1518.5 & 196.8 \\
\hline & rnd & 0.579 & 389 & 1373 & 287.0 & 0.38 & 12.3 & 1217.3 & 155.9 \\
\hline \multirow[t]{6}{*}{ Offset } & 1 & 0.690 & 119 & 2596 & 770.3 & 0.46 & 15.5 & 2326.6 & 269.3 \\
\hline & 2 & 0.594 & 295 & 2738 & 917.0 & 0.41 & 19.5 & 2407.0 & 331.4 \\
\hline & 4 & 0.433 & 848 & 3067 & 1094.0 & 0.32 & 26.5 & 2664.7 & 402.6 \\
\hline & 8 & 0.403 & 495 & 3166 & 1145.4 & 0.32 & 29.1 & 2729.3 & 436.4 \\
\hline & $\mathrm{r} 00$ & 0.220 & 867 & 3730 & 1222.7 & 0.33 & 32.5 & 3276.0 & 453.9 \\
\hline & rnd & 0.515 & 427 & 2930 & 948.8 & 0.43 & 20.6 & 2577.9 & 351.7 \\
\hline \multirow[t]{6}{*}{ BigLondon } & 1 & 0.600 & 225 & 2559 & 542.4 & 0.39 & 15.4 & 2317.8 & 241.7 \\
\hline & 2 & 0.514 & 418 & 2679 & 621.0 & 0.37 & 20.4 & 2374.1 & 305.0 \\
\hline & 4 & 0.450 & 662 & 2717 & 712.9 & 0.37 & 26.5 & 2341.5 & 375.2 \\
\hline & 8 & 0.457 & 639 & 2640 & 712.3 & 0.45 & 28.9 & 2257.1 & 383.4 \\
\hline & r00 & 0.257 & 942 & 3305 & 728.8 & 0.38 & 32.4 & 2879.3 & 425.2 \\
\hline & rnd & 0.490 & 453 & 2756 & 639.6 & 0.37 & 23.5 & 2409.9 & 346.6 \\
\hline
\end{tabular}

Table 4: Filter criteria available to the user.
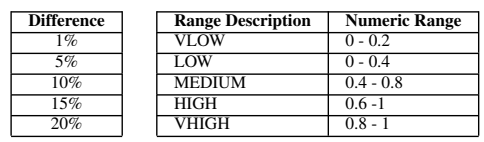

is now presented with a small number of solutions that they can evaluate. In many cases we note that tool includes some solutions that break the significance or range constraints, in such cases the penalty based fitness function may have included a solution that satisfies a constraint on one axis whilst only marginally breaking a constraint on another, thus the planner is presented with a set of solutions that attempt to meet their requirements, even when there are conflicts. In a small number of cases the tool returns 0 solutions due to there being no solutions in the underlying Pareto set which meet the criteria.

Figure 3 shows an example of the too in use, the upper plot is crowded and difficult for the planner to distinguish between individual solutions, the lower plot was generated by running the EA with the criteria as specified in the caption. Note that solutions presented are all within the ranges, the filter has attempted to eliminate solutions that violate the significance criteria. The lowest two solutions on the $\mathrm{CO}_{2}$ scale violate the significance constraint, but examination shows that they meet all of the other constraints, they have been included as the fitness function has traded off the penalty from the $\mathrm{CO}_{2}$ significance against the benefits accrued against the other criteria.

The EA has been incorporated in a Java Servlet, allowing it to be used interactively (see figure 2), source code for the tool may be found in the supplementary material accompanying this paper.

\section{CONCLUSIONS AND FURTHER WORK}

Within this paper we set out to answer two related questions, firstly can we generate Pareto sets of solutions that give the planner a meaningful choice and can we develop a mechanism to assist the

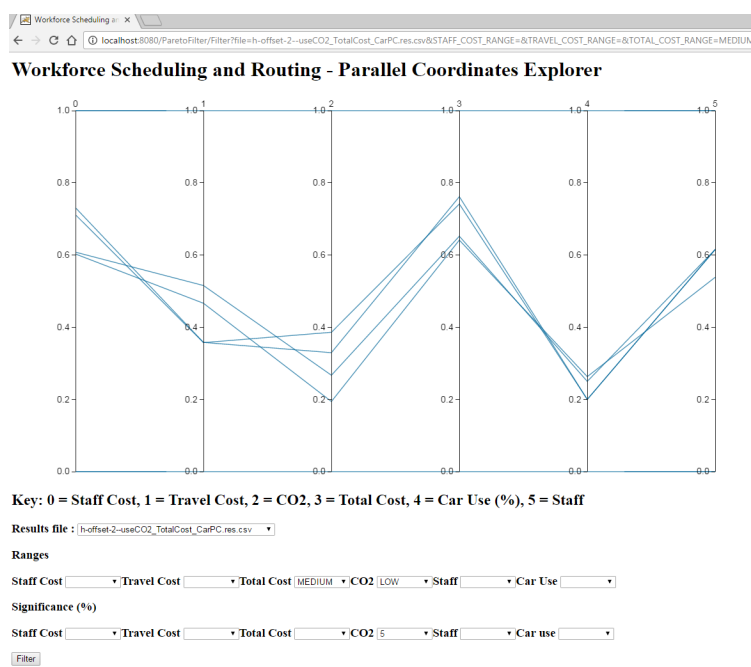

Figure 2: The EA incorporated into the Web based decision Support.

planner when making the choice of which solution to adopt? In order to answer these questions we propose and use a multi-objective optimisation technique to explore the sustainability of the mobility needed to provide a non-residential care service to a given number of patients. Emissions and impact on traffic are used as indicators of (social) sustainability. These indicators are compared to the cost to the service provider in terms of staff and travel cost. We base our study on synthetic populations of visits, located in London. This allows us using realistic values to determine paths, travel costs $\mathrm{CO} 2$ emissions, and staff costs. We do not identify single optimal solutions but we build the Pareto front of non-dominated solutions, which from the planning perspective represent solutions with a particular trade-off between objectives. We note that providing the Pareto front can give the service provider a greater control on its actions, above all if the Pareto sets are large as shown in table 2, 
Table 8: The before and after effects of running the evolvable filter with the criteria Travel Cost = MEDIUM, Total Cost = MEDIUM, CO2 Cost $=$ VLOW, Total Cost Significance $=1 \%$, CO2 Significance $=5 \%$. The figures shown are of the format before $/$ after .

\begin{tabular}{|l|c|c|c|c|c|c|c|c|c|}
\hline \multirow{2}{*}{ Problem } & \multicolumn{3}{|c|}{ Cardinality } & \multicolumn{2}{c|}{ Travel Cost } & \multicolumn{2}{c|}{ CO2 } & \multicolumn{2}{c|}{ Total Cost } \\
\cline { 2 - 9 } & Initial & After & Diff & Out of Range & Too Close & Out of Range & Too Close & Out of Range & Too Close \\
\hline lon-1 & 229 & 0 & $0.00 \%$ & $91 / 0$ & $0 / 0$ & $197 / 0$ & $211 / 0$ & $124 / 0$ & $0 / 0$ \\
\hline lon-2 & 315 & 1 & $0.32 \%$ & $315 / 0$ & $314 / 0$ & $264 / 0.2$ & $298 / 0$ & $168 / 0.8$ & $0 / 0$ \\
\hline lon-4 & 271 & 0 & $0.00 \%$ & $271 / 0$ & $270 / 0$ & $251 / 0$ & $258 / 0$ & $161 / 0$ & $0 / 0$ \\
\hline lon-8 & 461 & 3.2 & $0.69 \%$ & $461 / 0.2$ & $458 / 0$ & $411 / 0.3$ & $454 / 0.2$ & $247 / 0$ & $0 / 0$ \\
\hline lon-r00 & 511 & 5.5 & $1.08 \%$ & $511 / 0$ & $510 / 0$ & $404 / 0.6$ & $504 / 0.4$ & $271 / 0.1$ & $0 / 0$ \\
\hline lon-rnd & 338 & 0 & $0.00 \%$ & $338 / 0$ & $337 / 0$ & $313 / 0$ & $319 / 0$ & $180 / 0$ & $0 / 0$ \\
\hline blon-1 & 225 & 0 & $0.00 \%$ & $225 / 0$ & $222 / 0$ & $215 / 0$ & $209 / 0$ & $104 / 0$ & $0 / 0$ \\
\hline blon-2 & 418 & 0 & $0.00 \%$ & $418 / 0$ & $416 / 0$ & $367 / 0$ & $404 / 0$ & $220 / 0$ & $0 / 0$ \\
\hline blon-4 & 662 & 0 & $0.00 \%$ & $662 / 0$ & $660 / 0$ & $619 / 0$ & $651 / 0$ & $363 / 0$ & $0 / 0$ \\
\hline blon-8 & 639 & 1 & $0.16 \%$ & $639 / 0$ & $638 / 0$ & $612 / 0$ & $623 / 0$ & $348 / 1$ & $0 / 0$ \\
\hline blon-r00 & 942 & 1.3 & $0.14 \%$ & $942 / 0$ & $941 / 0$ & $829 / 0.6$ & $931 / 0.1$ & $441 / 0.3$ & $0 / 0$ \\
\hline blon-rnd & 453 & 0 & $0.00 \%$ & $453 / 0$ & $451 / 0$ & $437 / 0$ & $435 / 0$ & $203 / 0$ & $0 / 0$ \\
\hline cluster-1 & 174 & 0 & $0.00 \%$ & $174 / 0$ & $170 / 0$ & $172 / 0$ & $157 / 0$ & $79 / 0$ & $0 / 0$ \\
\hline cluster-2 & 279 & 2.6 & $0.93 \%$ & $279 / 0$ & $276 / 0$ & $263 / 1.6$ & $262 / 0$ & $133 / 0$ & $0 / 0$ \\
\hline cluster-4 & 267 & 8.4 & $3.15 \%$ & $267 / 0$ & $265 / 0$ & $154 / 1$ & $249 / 1.7$ & $141 / 0.1$ & $0 / 0$ \\
\hline cluster-8 & 467 & 6.4 & $1.37 \%$ & $467 / 0.2$ & $466 / 0$ & $346 / 1$ & $455 / 1$ & $262 / 0$ & $0 / 0$ \\
\hline cluster-r00 & 463 & 6.2 & $1.34 \%$ & $463 / 0.1$ & $462 / 0$ & $302 / 0.4$ & $454 / 0.9$ & $231 / 0.9$ & $0 / 0$ \\
\hline cluster-rnd & 389 & 5.5 & $1.41 \%$ & $389 / 0.3$ & $387 / 0$ & $326 / 0.3$ & $381 / 0.4$ & $197 / 0$ & $0 / 0$ \\
\hline offset-1 & 119 & 0 & $0.00 \%$ & $119 / 0$ & $116 / 0$ & $110 / 0$ & $93 / 0$ & $64 / 0$ & $0 / 0$ \\
\hline offset-2 & 295 & 1 & $0.34 \%$ & $295 / 0$ & $293 / 0$ & $286 / 0$ & $276 / 0$ & $161 / 0$ & $0 / 0$ \\
\hline offset-4 & 848 & 0 & $0.00 \%$ & $848 / 0$ & $846 / 0$ & $746 / 0$ & $835 / 0$ & $395 / 0$ & $0 / 0$ \\
\hline offset-8 & 495 & 0 & $0.00 \%$ & $495 / 0$ & $493 / 0$ & $379 / 0$ & $478 / 0$ & $222 / 0$ & $0 / 0$ \\
\hline offset-r00 & 867 & 0 & $0.00 \%$ & $867 / 0$ & $865 / 0$ & $657 / 0$ & $854 / 0$ & $412 / 0$ & $0 / 0$ \\
\hline offset-rnd & 427 & 0 & $0.00 \%$ & $427 / 0$ & $425 / 0$ & $312 / 0$ & $421 / 0$ & $288 / 0$ & $0 / 0$ \\
\hline
\end{tabular}

Table 5: The before and after effects of running the evolvable filter with the criteria Travel Cost $=$ Low, CO2 Cost $=$ VLOW, Travel Cost Significance $=5 \%, \mathrm{CO} 2$ Significance $=1 \%$. The figures shown are of the format before / after.

\begin{tabular}{|l|c|c|c|c|c|c|c|}
\hline \multirow{2}{*}{ Problem } & \multicolumn{3}{|c|}{ Cardinality } & \multicolumn{2}{c|}{ Travel Cost } & \multicolumn{2}{c|}{ CO2 } \\
\cline { 2 - 8 } & Initial & After & Diff & Out of Range & Too Close & Out of Range & Too Close \\
\hline lon-1 & 229 & 0 & $0.00 \%$ & $199 / 0$ & $225 / 0$ & $197 / 0$ & $211 / 0$ \\
\hline lon-2 & 315 & 0 & $0.00 \%$ & $242 / 0$ & $312 / 0$ & $264 / 0$ & $298 / 0$ \\
\hline lon-4 & 271 & 1 & $0.37 \%$ & $170 / 0$ & $268 / 0$ & $251 / 0$ & $258 / 0$ \\
\hline lon-8 & 461 & 4.7 & $1.02 \%$ & $305 / 0$ & $458 / 1.5$ & $411 / 0$ & $454 / 0.8$ \\
\hline lon-r00 & 511 & 2.6 & $0.51 \%$ & $337 / 0$ & $510 / 0.6$ & $404 / 0$ & $504 / 0.1$ \\
\hline lon-rnd & 338 & 3.8 & $1.12 \%$ & $161 / 0$ & $334 / 1.1$ & $313 / 0$ & $319 / 0.6$ \\
\hline blon-1 & 225 & 2 & $0.89 \%$ & $204 / 0$ & $222 / 0$ & $215 / 0$ & $209 / 0$ \\
\hline blon-2 & 418 & 5.9 & $1.41 \%$ & $280 / 0$ & $417 / 2.2$ & $367 / 0.2$ & $404 / 0.9$ \\
\hline blon-4 & 662 & 5.8 & $0.88 \%$ & $525 / 0$ & $660 / 1.7$ & $619 / 0$ & $651 / 1.6$ \\
\hline blon-8 & 639 & 4.1 & $0.64 \%$ & $443 / 0.6$ & $637 / 1.6$ & $612 / 0.1$ & $623 / 0.3$ \\
\hline blon-r00 & 942 & 4.7 & $0.50 \%$ & $585 / 0$ & $940 / 1.2$ & $829 / 0$ & $931 / 1.3$ \\
\hline blon-rnd & 453 & 5 & $1.10 \%$ & $366 / 0$ & $450 / 0.4$ & $437 / 0.3$ & $435 / 0.2$ \\
\hline cluster-1 & 174 & 1 & $0.57 \%$ & $161 / 0$ & $169 / 0$ & $172 / 0$ & $157 / 0$ \\
\hline cluster-2 & 279 & 2 & $0.72 \%$ & $204 / 0$ & $276 / 0$ & $263 / 0$ & $262 / 0.2$ \\
\hline cluster-4 & 267 & 5.8 & $2.17 \%$ & $169 / 0.5$ & $264 / 2.3$ & $154 / 0.1$ & $249 / 0.9$ \\
\hline cluster-8 & 467 & 2 & $0.43 \%$ & $357 / 0$ & $465 / 1$ & $346 / 0$ & $455 / 0$ \\
\hline cluster-r00 & 463 & 4.9 & $1.06 \%$ & $239 / 0.2$ & $460 / 1.2$ & $302 / 0$ & $454 / 0.4$ \\
\hline cluster-rnd & 389 & 5 & $1.29 \%$ & $262 / 0$ & $388 / 1.2$ & $326 / 0.1$ & $381 / 0.4$ \\
\hline offset-1 & 119 & 2 & $1.68 \%$ & $85 / 0$ & $114 / 0$ & $110 / 0$ & $93 / 0$ \\
\hline offset-2 & 295 & 1 & $0.34 \%$ & $245 / 0$ & $292 / 0$ & $286 / 0$ & $276 / 0$ \\
\hline offset-4 & 848 & 6.1 & $0.72 \%$ & $518 / 0$ & $847 / 2.1$ & $746 / 0$ & $835 / 0.8$ \\
\hline offset-8 & 495 & 5.9 & $1.19 \%$ & $290 / 0$ & $494 / 1.3$ & $379 / 0.6$ & $478 / 0.9$ \\
\hline offset-r00 & 867 & 6.4 & $0.74 \%$ & $315 / 0$ & $866 / 2.6$ & $657 / 0$ & $854 / 1.2$ \\
\hline offset-rnd & 427 & 7.4 & $1.73 \%$ & $263 / 0$ & $424 / 2.6$ & $312 / 0$ & $421 / 0.7$ \\
\hline
\end{tabular}

giving it flexibility in terms of finding solutions that address internal and social goals.

Although our approach can find large numbers of solutions, from the perspective of the agency, it is not necessarily the quantity of solutions that is of value, but the their attributes. We find that $\mathrm{CO} 2$ and total costs increase with the number of visits and depends on the characteristics of the provided service, with higher values corresponding to situations where visits must take place in shorter time windows and are arranged in clusters.

We note a significant drawback of the MOGA based approach to solving the problem is the large number of solutions from which the planner must make their final choice, we address this decision making problem by using a simple EA to filter solutions to produce a small subset of solutions quickly that the end planner can examine in more detail. The principle value of this tool is its' ability to
Table 6: The before and after effects of running the evolvable filter with the criteria Total Cost $=$ Low, $\mathrm{CO} 2$ Cost $=$ medium, Total Cost Significance $=5 \%, \mathrm{CO2}$ Significance $=1 \%$. The figures shown are of the format before / after.

\begin{tabular}{|l|c|c|c|c|c|c|c|}
\hline \multirow{2}{*}{ Problem } & \multicolumn{3}{|c|}{ Cardinality } & \multicolumn{2}{c|}{ Total Cost } & \multicolumn{2}{c|}{ CO2 } \\
\cline { 2 - 8 } & Initial & After & Diff & Out of Range & Too Close & Out of Range & Too Close \\
\hline lon-1 & 229 & 1.2 & $0.52 \%$ & $229 / / .2$ & $0 / 0$ & $138 / 0$ & $227 / 0$ \\
\hline lon-2 & 315 & 3.8 & $1.21 \%$ & $315 / 3.8$ & $0 / 0$ & $144 / 0$ & $313 / 0.5$ \\
\hline lon-4 & 271 & 3.3 & $1.22 \%$ & $271 / 3.3$ & $0 / 0$ & $169 / 0$ & $268 / 0.2$ \\
\hline lon-8 & 461 & 3.6 & $0.78 \%$ & $461 / 3.6$ & $0 / 0$ & $323 / 0$ & $460 / 0.5$ \\
\hline lon-r00 & 511 & 3.8 & $0.74 \%$ & $51 / 3.8$ & $0 / 0$ & $306 / 0.1$ & $510 / 0.5$ \\
\hline lon-rnd & 338 & 3 & $0.89 \%$ & $338 / 3$ & $0 / 0$ & $216 / 0$ & $336 / 0.4$ \\
\hline blon-1 & 225 & 2.1 & $0.93 \%$ & $225 / 2.1$ & $0 / 0$ & $201 / 0.1$ & $222 / 0$ \\
\hline blon-2 & 418 & 2.5 & $0.60 \%$ & $418 / 2.5$ & $0 / 0$ & $285 / 0$ & $417 / 0.3$ \\
\hline blon-4 & 662 & 3 & $0.45 \%$ & $662 / 3$ & $0 / 0$ & $482 / 0.2$ & $660 / 0.3$ \\
\hline blon-8 & 639 & 3.5 & $0.55 \%$ & $639 / 3.5$ & $0 / 0$ & $537 / 0.3$ & $638 / 1$ \\
\hline blon-r00 & 942 & 3.4 & $0.36 \%$ & $942 / 3.4$ & $0 / 0$ & $604 / 0$ & $941 / 0.3$ \\
\hline blon-rnd & 453 & 3.4 & $0.75 \%$ & $453 / 3.4$ & $0 / 0$ & $324 / 0.2$ & $452 / 0.7$ \\
\hline cluster-1 & 174 & 2.9 & $1.67 \%$ & $174 / 2.9$ & $0 / 0$ & $94 / 0$ & $167 / 0.4$ \\
\hline cluster-2 & 279 & 3.9 & $1.40 \%$ & $279 / 3.9$ & $0 / 0$ & $164 / 0.4$ & $276 / 0.7$ \\
\hline cluster-4 & 267 & 3.7 & $1.39 \%$ & $267 / 3.7$ & $0 / 0$ & $68 / 0$ & $266 / 0.2$ \\
\hline cluster-8 & 467 & 4 & $0.86 \%$ & $467 / 4$ & $0 / 0$ & $119 / 0$ & $464 / 0.3$ \\
\hline cluster-r00 & 463 & 3.5 & $0.76 \%$ & $463 / 3.5$ & $0 / 0$ & $151 / 0$ & $460 / 0.3$ \\
\hline cluster-rnd & 389 & 3.9 & $1.00 \%$ & $389 / 3.9$ & $0 / 0$ & $206 / 0.2$ & $387 / 0.8$ \\
\hline offset-1 & 119 & 2.8 & $2.35 \%$ & $119 / 2.8$ & $0 / 0$ & $91 / 0$ & $115 / 0.3$ \\
\hline offset-2 & 295 & 2 & $0.68 \%$ & $295 / 2$ & $0 / 0$ & $229 / 0$ & $293 / 0$ \\
\hline offset-4 & 848 & 2.3 & $0.27 \%$ & $848 / 2.3$ & $0 / 0$ & $380 / 0.2$ & $847 / 0.5$ \\
\hline offset-8 & 495 & 1.7 & $0.34 \%$ & $495 / 1.7$ & $0 / 0$ & $282 / 0$ & $494 / 0.3$ \\
\hline offset-r00 & 867 & 1.7 & $0.20 \%$ & $867 / 1.7$ & $0 / 0$ & $384 / 0.2$ & $866 / 0$ \\
\hline offset-rnd & 427 & 2 & $0.47 \%$ & $427 / 2$ & $0 / 0$ & $224 / 0$ & $425 / 0.1$ \\
\hline
\end{tabular}

apply custom filter criteria quickly in order to support the decision making process being undertaken by the end user. The filter also has the ability to work with any of the problem criteria that are being made available (e.g. total cost, staff cost, travel cost, $\mathrm{CO}_{2}$, car use etc.) even when that criteria is not part of the optimisation criteria. Future developments could include allowing the user to specify more complex criteria (e.g. specific features within the solution that are desirable or undesirable to a certain degree).

Future developments would include more constrained staffing scenarios, with a limited number of staff and different skills among employees so that certain employees can carry out only certain types of visits as well as considering travel modes such car sharing.

\section{REFERENCES}

[1] Kris Braekers, Richard F. Hartl, Sophie N. Parragh, and Fabien Tricoire. 2016. A bi-objective home care scheduling problem: Analyzing the trade-off between 
Table 7: The before and after effects of running the evolvable filter with the criteria Travel Cost $=$ MEDIUM, CO2 Cost $=$ LOW, Travel Cost Significance $=10 \%, \mathrm{CO} 2$ Significance $=5 \%$. The figures shown are of the format before / after.

\begin{tabular}{|l|c|c|c|c|c|c|c|}
\hline \multirow{2}{*}{ Problem } & \multicolumn{3}{|c|}{ Cardinality } & \multicolumn{2}{c|}{ Travel Cost } & \multicolumn{2}{c|}{ CO2 } \\
\cline { 2 - 8 } & Initial & After & Diff & Out of Range & Too Close & Out of Range & Too Close \\
\hline lon-1 & 229 & 4.4 & $1.92 \%$ & $229 / 4.4$ & $0 / 0$ & $141 / 0.5$ & $211 / 0$ \\
\hline lon-2 & 315 & 6.8 & $2.16 \%$ & $315 / 6.8$ & $0 / 0$ & $200 / 0$ & $298 / 1$ \\
\hline lon-4 & 271 & 7.4 & $2.73 \%$ & $271 / 7.4$ & $0 / 0$ & $125 / 0$ & $258 / 0.7$ \\
\hline lon-8 & 461 & 6.8 & $1.48 \%$ & $461 / 6.8$ & $0 / 0$ & $168 / 0.1$ & $454 / 0.7$ \\
\hline lon-r00 & 511 & 6.9 & $1.35 \%$ & $511 / 6.9$ & $0 / 0$ & $257 / 0.1$ & $504 / 0.1$ \\
\hline lon-rnd & 338 & 7.1 & $2.10 \%$ & $338 / 7.1$ & $0 / 0$ & $141 / 0$ & $319 / 0.7$ \\
\hline blon-1 & 225 & 3.2 & $1.42 \%$ & $225 / 3.2$ & $0 / 0$ & $49 / 0$ & $209 / 0.2$ \\
\hline blon-2 & 418 & 5.4 & $1.29 \%$ & $418 / 5.4$ & $0 / 0$ & $177 / 0$ & $404 / 0.4$ \\
\hline blon-4 & 662 & 6 & $0.91 \%$ & $662 / 6$ & $0 / 0$ & $220 / 0$ & $651 / 0.4$ \\
\hline blon-8 & 639 & 5.9 & $0.92 \%$ & $639 / 5.9$ & $0 / 0$ & $176 / 0$ & $623 / 0.2$ \\
\hline blon-r00 & 942 & 6.6 & $0.70 \%$ & $942 / 6.6$ & $0 / 0$ & $380 / 0$ & $931 / 0.3$ \\
\hline blon-rnd & 453 & 5 & $1.10 \%$ & $453 / 5$ & $0 / 0$ & $155 / 0$ & $435 / 0.3$ \\
\hline cluster-1 & 174 & 3 & $1.72 \%$ & $174 / 3$ & $0 / 0$ & $86 / 0$ & $157 / 0.3$ \\
\hline cluster-2 & 279 & 7.1 & $2.54 \%$ & $279 / 7.1$ & $0 / 0$ & $133 / 0$ & $262 / 0.5$ \\
\hline cluster-4 & 267 & 5.1 & $1.91 \%$ & $267 / 5.1$ & $0 / 0$ & $214 / 0$ & $249 / 0.3$ \\
\hline cluster-8 & 467 & 5.8 & $1.24 \%$ & $467 / 5.8$ & $0 / 0$ & $353 / 0$ & $455 / 1$ \\
\hline cluster-r00 & 463 & 5.4 & $1.17 \%$ & $463 / 5.4$ & $0 / 0$ & $318 / 0.2$ & $454 / 0.4$ \\
\hline cluster-rnd & 389 & 7.1 & $1.83 \%$ & $389 / 7.1$ & $0 / 0$ & $194 / 0$ & $381 / 0.8$ \\
\hline offset-1 & 119 & 3.7 & $3.11 \%$ & $119 / 3.7$ & $0 / 0$ & $42 / 0$ & $93 / 0$ \\
\hline offset-2 & 295 & 3.3 & $1.12 \%$ & $295 / 3.3$ & $0 / 0$ & $85 / 0$ & $276 / 0.1$ \\
\hline offset-4 & 848 & 5.1 & $0.60 \%$ & $848 / 5.1$ & $0 / 0$ & $499 / 0$ & $835 / 0.4$ \\
\hline offset-8 & 495 & 6.1 & $1.23 \%$ & $495 / 6.1$ & $0 / 0$ & $254 / 0.8$ & $478 / 0.4$ \\
\hline offset-r00 & 867 & 4.6 & $0.53 \%$ & $867 / 4.6$ & $0 / 0$ & $510 / 0$ & $854 / 0$ \\
\hline offset-rnd & 427 & 4.3 & $1.01 \%$ & $427 / 4.3$ & $0 / 0$ & $225 / 0$ & $421 / 0$ \\
\hline
\end{tabular}

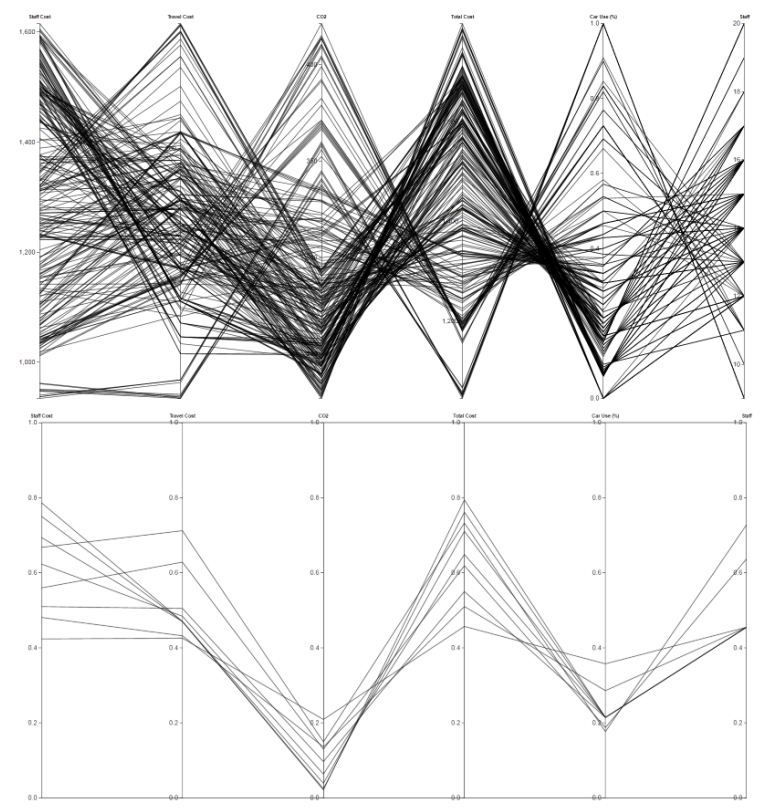

Figure 3: An example of the filter in use. In this case the initial Pareto front (top) was generated from the problem Cluster-4 using Total Cost, $\mathrm{CO}_{2}$ and car use as the optimisation criteria. The lower plot was generated after aplying the filter with the criteria Travel Cost $=$ MEDIUM, Total Cost $=$ MEDIUM, $\mathbf{C O 2}$ Cost $=$ VLOW, Total Cost Significance $=1 \%$, CO2 Significance $=5 \%$.

costs and client inconvenience. European Journal of Operational Research 248 2 (2016), 428 - 443. DOI : http://dx.doi.org/10.1016/j.ejor.2015.07.028

[2] J. Arturo Castillo-Salazar, Dario Landa-Silva, and Rong Qu. 2016. Workforce scheduling and routing problems: literature survey and computational study. Annals of Operations Research 239, 1 (2016), 39-67. DOI : http://dx.doi.org/10 1007/s10479-014-1687-2
[3] Landa-Silva D. Castro-Gutierrez, J. and J Moreno Perez. 2011. Nature of realworld multi-objective vehicle routing with evolutionary algorithms. International Conference on Systems, Man, and Cybernetics (2011), 257-264. DOI : http: //dx.doi.org/10.1109/ICSMC.2011.6083675

[4] Rommert Dekker, Jacqueline Bloemhof, and Ioannis Mallidis. 2012. Operations Research for green logistics An overview of aspects, issues, contributions and challenges. European Journal of Operational Research 219, 3 (2012), 671 - 679. DOI : http://dx.doi.org/10.1016/j.ejor.2011.11.010 Feature Clusters.

[5] Dragan Pamuar Goran irovi. 2014. Green logistic vehicle routing problem: Routing light delivery vehicles in urban areas using a neuro-fuzzy model. Expert Systems with Applications 41, 9 (2014), 4245-4258. DOI : http://dx.doi.org/10. 1016/j.eswa.2014.01.005

[6] National Health Service Employers. 2016. Millage Allowances. (2016). http://www.nhsemployers.org/your-workforce/pay-and-reward/ nhs-terms-and-conditions/nhs-terms-and-conditions-of-service-handbook/ mileage-allowances.

[7] Transport for London. 2009. Travel in London: Key trends and develpments. Technical Report. Transport for London.

[8] Transport for London. 2015. Transport for London Unified API. (2015). https: //api.tfl.gov.uk/

[9] OpenStreetMap Foundation. 2014. http://www.openstreetmap.org. (2014). http: //www.openstreetmap.org

[10] Nissen V Gunther M. 2012. Application of particle swarm optimization to the british telecom workforce scheduling problem. Proceedings of the 9th International Conference on the Practice and Theory of Automated Timetabling (PATAT 2012), Son, Norway (2012).

[11] M. Haklay and P. Weber. 2008. OpenStreetMap: User-Generated Street Maps. Pervasive Computing, IEEE 7, 4 (Oct 2008), 12-18. DOI : http://dx.doi.org/10. 1109/MPRV.2008.80

[12] Gerhard Hiermann, Matthias Prandtstetter, Andrea Rendl, Jakob Puchinger, and Günther R. Raidl. 2015. Metaheuristics for solving a multimodal home-healthcare scheduling problem. Central European Journal of Operations Research 23, 1 (2015), 89-113. DOI : http://dx.doi.org/10.1007/s10100-013-0305-8

[13] A. Inselberg. 1997. Multidimensional Detective. In Proceedings of the 1997 IEEE Symposium on Information Visualization (InfoVis '97) (INFOVIS '97). IEEE Computer Society, Washington, DC, USA, 100-. http://dl.acm.org/citation.cfm? id $=857188.857631$

[14] A. Inselberg. 2009. Parallel Coordinates. Visual MultidimensionalGeometry and its applications. Springer

[15] G irovi, D Pamuar, and D Boani. 2014. Green logistic vehicle routing problem: Routing light delivery vehicles in urban areas using a neuro-fuzzy model. Expert Systems with Applications (2014), 4245 - 4258.

[16] P Karich. 2014. GraphHopper. https://graphhopper.com/. (2014). https: //graphhopper.com/

[17] Smet P.-Verbeeck K. Misir, M. and G. Vanden Berghe. 2011. Security personnel routing and rostering: a hyper- heuristic approach. Proceedings of the $3 \mathrm{rd}$ International Conference on Applied Operational Research, ICAORII (2011).

[18] Payscale.com. 2016. Social Worker Salary (UK). (2016). http://www.payscale. $\mathrm{com} /$ research/UK/Job=Social_Worker/Salary

[19] Jiani Qian and Richard Eglese. 2016. Fuel emissions optimization in vehicle routing problems with time-varying speeds. European Journal of Operational Research 248, 3 (2016), 840 - 848. DOI : http://dx.doi.org/10.1016/j.ejor.2015. 09.009

[20] Matias Sevel Rasmussen, Tor Justesen, Anders Dohn, and Jesper Larsen. 2010. The Home Care Crew Scheduling Problem:: Preference-Based Visit Clustering and Temporal Dependencies. DTU Management.

[21] M. M. Solomon. 1987. Algorithms for the Vehicle Routing and Scheduling Problems with Time Window Constraints. Oper. Res. 35, 2 (April 1987), 254-265. DOI : http://dx.doi.org/10.1287/opre.35.2.254

[22] Neil Urquhart. 2017. Online ParetoEA Decision Support Tool. (2017). http: //socoptimisation.napier.ac.uk:8080/ParetoFilter/Filter

[23] Neil Urquhart and Emma Hart. 2015. Creating Optimised Employee Travel Plans.. In Proceedings of the The 11th edition of the International Conference on Evolutionary and Deterministic Methods for Design, Optimization and Control with Applications to Industrial and Societal Problems.

[24] Neil B. Urquhart, Emma Hart, and Alistair Judson. 2015. Multi-Modal Employee Routing with Time Windows in an Urban Environment. In Proceedings of the Companion Publication of the 2015 Annual Conference on Genetic and Evolutionary Computation (GECCO Companion '15). ACM, New York, NY, USA, 1503-1504. DOI : http://dx.doi.org/10.1145/2739482.2764649

[25] Eckart Zitzler, Dimo Brockhoff, and Lothar Thiele. 2007. The Hypervolume Indicator Revisited: On the Design of Pareto-compliant Indicators Via Weighted Integration. Springer Berlin Heidelberg, Berlin, Heidelberg, 862-876. DOI : http://dx.doi.org/10.1007/978-3-540-70928-2_64 